\title{
Spontaneous regression and near disappearance of a calcified herniated thoracic disc in a 44-year-old male: illustrative case
}

\author{
Andrew Utter, MD, David Cavanaugh, MD, Kelly F. Van Schouwen, MS, Madison Mascagni, Madeline Walker, BS, and \\ Marcus Stone, $\mathrm{PhD}$
}

Spine Institute of Louisiana, Shreveport, Louisiana

BACKGROUND Herniation of an intervertebral disc (IVD) is found predominantly in the lumbar and cervical spine of both children and adults, but herniated IVDs of the thoracic spine are a rare occurrence. However, approximately $40 \%$ of herniated thoracic disc cases are calcified. Approximately $0.65 \%$ of all spinal herniations are calcified herniated thoracic discs (CHTDs). CHTDs can be treated conservatively or invasively, depending on the symptoms and degree of neurological deficit present.

OBSERVATIONS The authors report a 44-year-old male with near complete reabsorption and disappearance of a CHTD. A review of the available literature indicates that there are only seven adult patients in whom this phenomenon has been reported.

LESSONS Determining the best form of invasive treatment is a challenge for surgeons given the complexity of this condition. While the disappearance of calcified herniated discs of the lumbar and cervical spine has been reported, reports of the regression of CHTDs are rare. The disappearance of CHTDs is more commonly reported in children who undergo conservative treatment, while surgery is reserved for children who experience progressive pain and neurological deficit. Given the success of conservative treatment of CHTDs in children, conservative treatment methods should be considered when treating mildly symptomatic adults.

https://thejns.org/doi/abs/10.3171/CASE2130

KEYWORDS intervertebral disc; disc herniation; thoracic disc extrusion; calcified thoracic disc

Herniation of an intervertebral disc (IVD) is found predominantly in the lumbar and cervical spine, but herniated IVDs of the thoracic spine are a rare occurrence. However, approximately $40 \%$ of herniated thoracic disc cases are calcified. ${ }^{1-3}$ The calcified nucleus pulposus of an IVD can herniate into the spinal canal, which is considered to be an exceedingly rare complication of this condition. ${ }^{4}$ Calcified herniated thoracic discs (CHTDs) account for less than $1 \%$ of all spinal herniations because herniation of a thoracic disc is much more rare than cervical or lumbar disc herniation. ${ }^{1,3,5}$ In cases in which an adult presents with a CHTD, research suggests little chance of spontaneous regression, and surgical treatment is usually required. ${ }^{6}$ The spontaneous regression of a calcified herniated disc in the lumbar or cervical spine has been commonly reported. However, the spontaneous regression of a
CHTD is an extremely unusual occurrence. Such spontaneous regression is more frequently seen in children, with few reports of this phenomenon occurring in adults. ${ }^{5}$ A review of the available literature indicates that there are reports of only seven adult patients with a CHTD that spontaneously resolved. Three patients were reported in two French-language articles, and four patients were reported in four English-language articles. ${ }^{1,5,7-10}$

\section{Illustrative Case}

A 44-year-old male on active military duty presented to our clinic with thoracic back pain. The onset of pain was sudden and occurred while he was sitting at his desk chair 5 months before his initial visit in 2015. The patient described the occurrence as "an odd feeling, almost like the feeling of having a rock in your shoe." Four months after

ABBREVIATIONS CHTD = calcified herniated thoracic disc; CT = computed tomography; IVD = intervertebral disc; MRI = magnetic resonance imaging INCLUDE WHEN CITING Published May 10, 2021; DOI: 10.3171/CASE2130.

SUBMITTED January 13, 2021. ACCEPTED February 14, 2021.

(C) 2021 The authors, CC BY-NC-ND 4.0 (http://creativecommons.org/licenses/by-nc-nd/4.0/). 

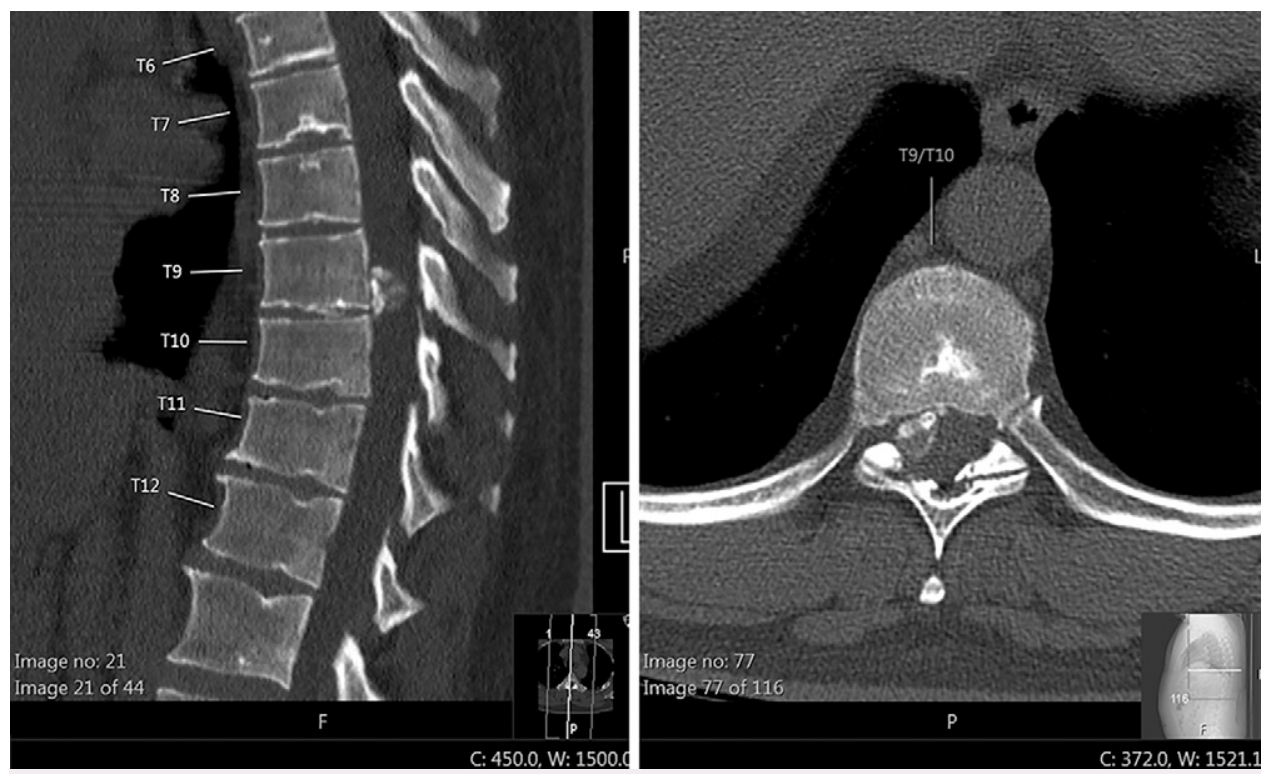

FIG. 1. Sagittal (left) and axial (right) CT views at initial presentation.

the initial onset of pain, the patient was evaluated by military physicians and prescribed physical therapy. In physical therapy, the patient performed exercises such as press-ups, which made his back pain worse. He received ketorolac tromethamine shots and steroid injections in his arm and buttock, which he reported did not help with his pain. During his initial visit to our clinic, the patient denied any bowel or bladder incontinence, numbness and weakness in the lower extremities, or changes in balance. On physical examination, the patient demonstrated a normal gait. His strength was $5 / 5$ in the lower extremities, his reflexes were intact, and his sensation showed some allodynia in his right flank area.

At his initial visit in our clinic, the patient brought a magnetic resonance imaging (MRI) study without contrast of the thoracic and lumbar spine that had been performed in 2015. The MRI showed an extradural mass, initially thought to be a soft thoracic disc herniation, that measured $1.3 \mathrm{~cm}$ at the T9-10 level on the right side. The mass demonstrated cord compression. Two weeks later, MRI with and without contrast was performed and confirmed the herniation at T9-10. For treatment, a right T9-10 discectomy was discussed with the patient, and a preoperative computed tomography (CT) scan was obtained. The CT without contrast of the thoracic spine revealed a large CHTD at T9-10 (Fig. 1). Because of the increased surgical risks associated with treating a CHTD, the patient elected to forgo the surgery and opted for conservative treatment. A permanent work restriction was recommended to the patient, which consisted of a lifting restriction of 20 pounds and no excessive bending or extending of his back. The patient continued with pain management and physical therapy.

Upon his return to the clinic in early 2016, the patient reported that his pain had been gradually decreasing and that physical therapy still aggravated the pain in his midback. He was advised to continue conservative treatment given his improved condition. Four months later, the patient reported some intermittent numbness of his feet and stated that his pain had gotten "a little bit worse" but was manageable. The patient reported that his pain was fairly severe for about 4 to 5 days per month, particularly after performing activities such as yard work. The pain was relieved with medications (ketorolac tromethamine; Percocet, Endo Pharmaceuticals Inc.) and when lying down. His strength, sensation, and reflexes remained intact. He denied any bowel or bladder incontinence or changes in balance.

Almost 2 years after the patient's initial presentation, he returned to the clinic with complaints of continued thoracic pain as well as new-onset cervical pain. The patient stated that while his thoracic pain was not constant, it occasionally flared up, requiring medication. Follow-up MRI without contrast of the cervical, thoracic, and lumbar spine was performed. The thoracic spine MRI showed the central spinal canal and neural foramina to be intact throughout with disappearance of the CHTD at T9-10. The patient consented to a CT scan to confirm the disappearance of the CHTD. Upon review of the CT, it was confirmed that the CHTD showed nearly complete resolution over an approximately 2-year period (Fig. 2). Although the CHTD mostly disappeared, the patient had ongoing episodic thoracic spine pain. He continued to deny any bowel or bladder incontinence, and physical examination found no numbness and weakness in the lower extremities or changes in balance.

\section{Discussion \\ Observations}

Although intervertebral calcifications are an overall rare occurrence, the reported cases suggest they are found more commonly in adult patients with degenerating discs. ${ }^{5,11}$ It is an extremely rare occurrence for a CHTD to resolve and disappear in an adult patient. A review of the literature revealed seven patient reports, three written in French and four in English, of CHTDs disappearing in adults. $^{1,5,7-10}$ All seven of the reported patients were treated conservatively, and each showed complete resolution of the CHTD. Oligane et al. reported a 40-year-old woman who presented with unusual abdominal cramping and lower back pain and later 

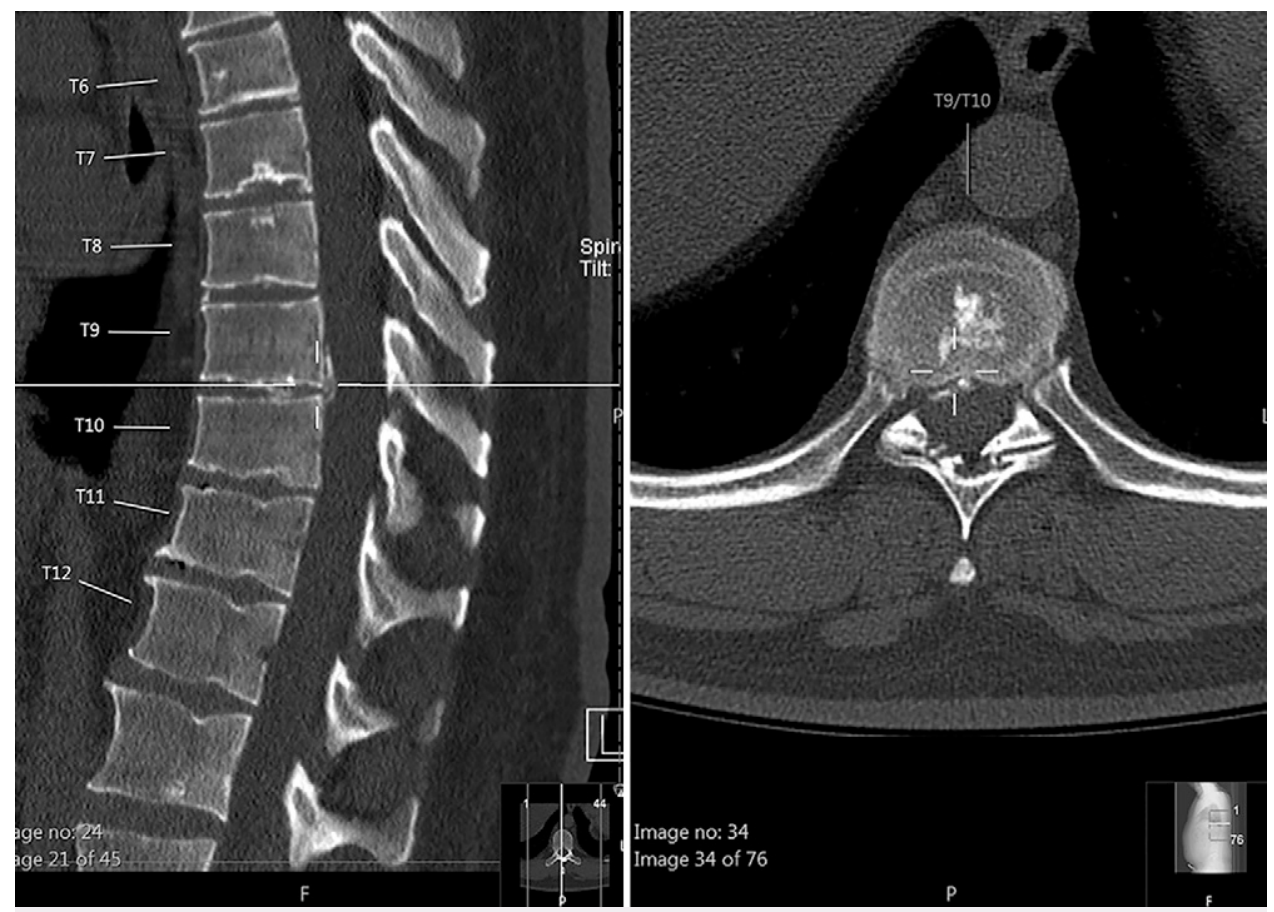

FIG. 2. Sagittal (left) and axial (right) CT views at last presentation.

demonstrated a large CHTD at T10-11. Follow-up MRI performed 8 months after her initial visit revealed complete regression of the CHTD. ${ }^{5}$ Ahmad et al. reported a 38 -year-old female with thalassemia who, over an approximately 2-year period, demonstrated spontaneous resolution of a CHTD at T6-7. ${ }^{7}$ Piccirilli et al. reported spontaneous regression of a CHTD in a 36-year-old female over 11 months. ${ }^{9}$ Sari et al. reported a 38-year-old male with calcifications of T7-8 and T8-9 IVDs with a CHTD at T7-8. Over a 5-month period, the CHTD at T7-8 spontaneously resolved, while the IVD calcification at T8-9 remained the same. ${ }^{10}$

In each of the cases described above, the patients experienced a gradual regression of their symptoms over the time frame in which their CHTDs resolved. For treatment, two of the patients underwent physical therapy for their associated symptoms. ${ }^{5,10}$ According to Oligane et al., the patient's abdominal cramping and back pain became aggravated after undergoing physical therapy. ${ }^{5}$ Similarly, our patient presented with aggravated thoracic back pain after undergoing physical therapy. In the case of Sari et al., the patient underwent analgesic and nonsteroidal anti-inflammatory drug treatment and 15 physical therapy sessions, which resulted in complete regression of his symptoms and removed any restrictions from his daily living activities. ${ }^{10}$ It is apparent that there is no set guideline on conservative treatment of CHTD, with varied results across treatments and patients. Similar to the challenge of surgical intervention for treating such conditions, each case is unique and requires an in-depth assessment before determining the best form of treatment.

The spontaneous disappearance of CHTDs is more commonly seen in children. A CHTD in a child is often resolved with conservative treatment, whereas surgical intervention is often required for adults. ${ }^{11}$ Conservative treatment for this condition consists of bed rest, wearing a brace or collar, lifestyle adjustments, and nonsteroidal anti-inflammatory drugs. For instance, a 12-year-old female was treated conservatively for CHTDs at T7-8 and T11-12. Her midback pain diminished within 3 weeks, and a CT scan obtained at her 10-month follow-up revealed complete resolution of both $\mathrm{CHTDs} .{ }^{4}$ Surgery is often reserved for children with uncontrollable pain or progressive neurological deficit. ${ }^{4,5}$ Perhaps because of the remarkable results seen in children, conservative treatment should be considered before surgical intervention when treating a CHTD in an adult.

When patients with CHTDs present with severe pain and neurological deficit, surgery is often the recommended form of treatment. ${ }^{11}$ Determining the best form of surgical treatment is a challenge for surgeons because of the rarity and complexity of this condition. ${ }^{1,5}$ While there are few reports on how to treat CHTDs surgically, posterior decompression and segmental instrumentation with interbody fusion is the most commonly reported form of treatment. In a study performed to assess the outcomes for patients who underwent such treatment, the overall results showed significant postoperative improvement and recovery. ${ }^{12}$ However, according to a study performed by Al-Mahfoudh et al., a trench vertebrectomy via a thoracotomy allows safer identification of the compressed segment before a discectomy is performed. This proposed surgical method had an $83.3 \%$ subject improvement following the treatment. ${ }^{13}$ Overall, there is significant debate on which surgical option is best for this complex condition.

The cause of our patient's spontaneous disappearance of a CHTD is unknown. Various hypotheses have been proposed for the mechanism of spontaneous regression of CHTDs. Oligane et al. suggested "regression due to dehydration and shrinkage of the herniated nucleus pulposus; retraction of the herniated disk into the intervertebral space; and an inflammatory reaction with enzymatic degradation and phagocytosis." ${ }^{5}$ It is possible that our patient's persistent physical therapy 
induced an inflammatory response that increased his blood circulation, which could have resulted in degradation of the CHTD.,9

\section{Lessons}

We report an unusual case of a CHTD in a 44-year-old male resolving without surgical intervention. The gold standard of treatment for CHTDs remains controversial. The rare reports of disappearing CHTDs support the proposal of clinical and radiological surveillance in patients with mild symptoms before putting them at risk of surgical intervention. ${ }^{2,14}$ Perhaps conservative treatment over a period of time should be considered when assessing safe treatment options for CHTDs.

\section{References}

1. Eap C, Bennis S, Blauwblomme T, et al. Spontaneous resorption of thoracic calcified disc herniation: report of two cases and review of the literature. Article in French. Neurochirurgie. 2012;58(6):353-357.

2. Girodias JB, Azouz EM, Marton D. Intervertebral disk space calcification. A report of 51 children with a review of the literature. Pediatr Radiol. 1991;21(8):541-546.

3. Court C, Mansour E, Bouthors C. Thoracic disc herniation: surgical treatment. Orthop Traumatol Surg Res. 2018;104(1S):S31-S40.

4. Babashahi A, Taheri M, Rabiee P. Spontaneous resolution of symptomatic thoracic spine calcified disc herniation: a case report and literature review. Iran J Med Sci. 2019;44(3):251-256.

5. Oligane H, Rongo J, Agarwal V, Branstetter BF IV. Spontaneous regression of a large calcified thoracic disk extrusion. Skeletal Radiol. 2018;47(8):1177-1182.

6. Morandi X, Crovetto N, Carsin-Nicol B, et al. Spontaneous disappearance of a thoracic disc hernia. Article in French. Neurochirurgie. 1999;45(2):155-159.

7. Ahmad FU, Schallert E, Bregy A, et al. Disappearing large calcified thoracic disc herniation in a patient with thalassaemia. BMJ Case Rep. 2016;2016:bcr2015213166.

8. Coevoet V, Benoudiba F, Lignières C, et al. Spontaneous and complete regression in MRI of thoracic disk herniation. Article in French. J Radiol. 1997;78(2):149-151.

9. Piccirilli M, Lapadula G, Caporlingua F, et al. Spontaneous regression of a thoracic calcified disc herniation in a young female: a case report and literature review. Clin Neurol Neurosurg. 2012; 114(6):779-781.

10. Sari $H$, Misirlioglu TO, Palamar D. Regression of a symptomatic thoracic disc herniation with a calcified intervertebral disc component. Acta Orthop Traumatol Turc. 2016;50(6):698-701.

11. Bajard X, Renault $F$, Benharrats $T$, et al. Intervertebral disc calcification with neurological symptoms in children: report of conservative treatment in two cases. Childs Nerv Syst. 2010;26(7):973-978.

12. Zhuang QS, Lun DX, Xu ZW, et al. Surgical treatment for central calcified thoracic disk herniation: a novel L-shaped osteotome. Orthopedics. 2015;38(9):e794-e798.

13. Al-Mahfoudh R, Mitchell PS, Wilby M, et al. Management of giant calcified thoracic disks and description of the trench vertebrectomy technique. Global Spine J. 2016;6(6):584-591.

14. Ho C, Chang S, Fulkerson D, Smith J. Children presenting with calcified disc herniation: a self-limiting process. J Radiol Case Rep. 2012;6(10):11-19.

\section{Disclosures}

Ms. Van Schouwen reports other support from Surgalign, Cerapedics, $\mathrm{BiO}_{2}$, Synergy Disc, and SI-BONE outside the submitted work. Dr. Stone reports receiving personal fees from Spineology and Organogenesis, other support from Simplify Medical and Zimmer Biomet, and grants from Wright Medical outside the submitted work.

\section{Author Contributions}

Conception and design: Stone, Utter, Cavanaugh, Van Schouwen. Acquisition of data: Stone. Analysis and interpretation of data: Stone, Cavanaugh. Drafting the article: Stone, Van Schouwen, Mascagni, Walker. Critically revising the article: Stone, Cavanaugh, Van Schouwen, Mascagni. Reviewed submitted version of manuscript: Stone, Utter, Cavanaugh, Van Schouwen, Mascagni. Approved the final version of the manuscript on behalf of all authors: Stone. Administrative/technical/material support: Cavanaugh, Walker. Study supervision: Utter.

\section{Correspondence}

Marcus Stone: Spine Institute of Louisiana, Shreveport, LA. mstone@ louisianaspine.org. 